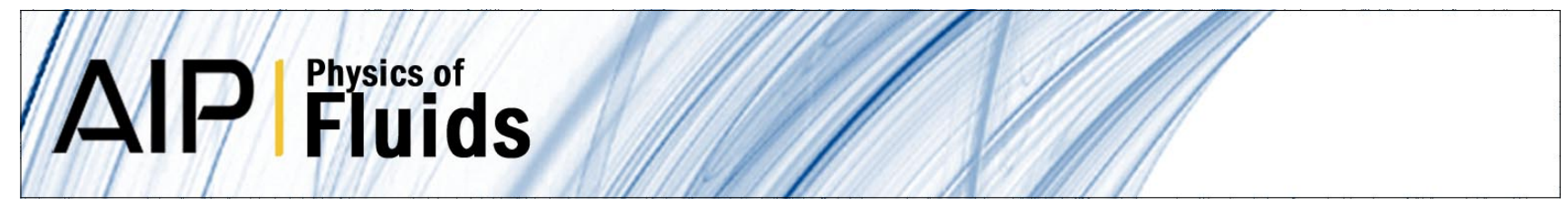

\title{
On Lagrangian single-particle statistics
}

Gregory Falkovich, Haitao Xu, Alain Pumir, Eberhard Bodenschatz, Luca Biferale et al.

Citation: Phys. Fluids 24, 055102 (2012); doi: 10.1063/1.4711397

View online: http://dx.doi.org/10.1063/1.4711397

View Table of Contents: http://pof.aip.org/resource/1/PHFLE6/v24/i5

Published by the American Institute of Physics.

\section{Related Articles}

The length distribution of streamline segments in homogeneous isotropic decaying turbulence Phys. Fluids 24, 045104 (2012)

Conditional vorticity budget of coherent and incoherent flow contributions in fully developed homogeneous isotropic turbulence

Phys. Fluids 24, 035108 (2012)

Near-field investigation of turbulence produced by multi-scale grids

Phys. Fluids 24, 035103 (2012)

Reynolds number effect on the velocity increment skewness in isotropic turbulence

Phys. Fluids 24, 015108 (2012)

Spectral approach to finite Reynolds number effects on Kolmogorov's 4/5 law in isotropic turbulence Phys. Fluids 24, 015107 (2012)

\section{Additional information on Phys. Fluids}

Journal Homepage: http://pof.aip.org/

Journal Information: http://pof.aip.org/about/about_the_journal

Top downloads: http://pof.aip.org/features/most_downloaded

Information for Authors: http://pof.aip.org/authors

\section{ADVERTISEMENT}

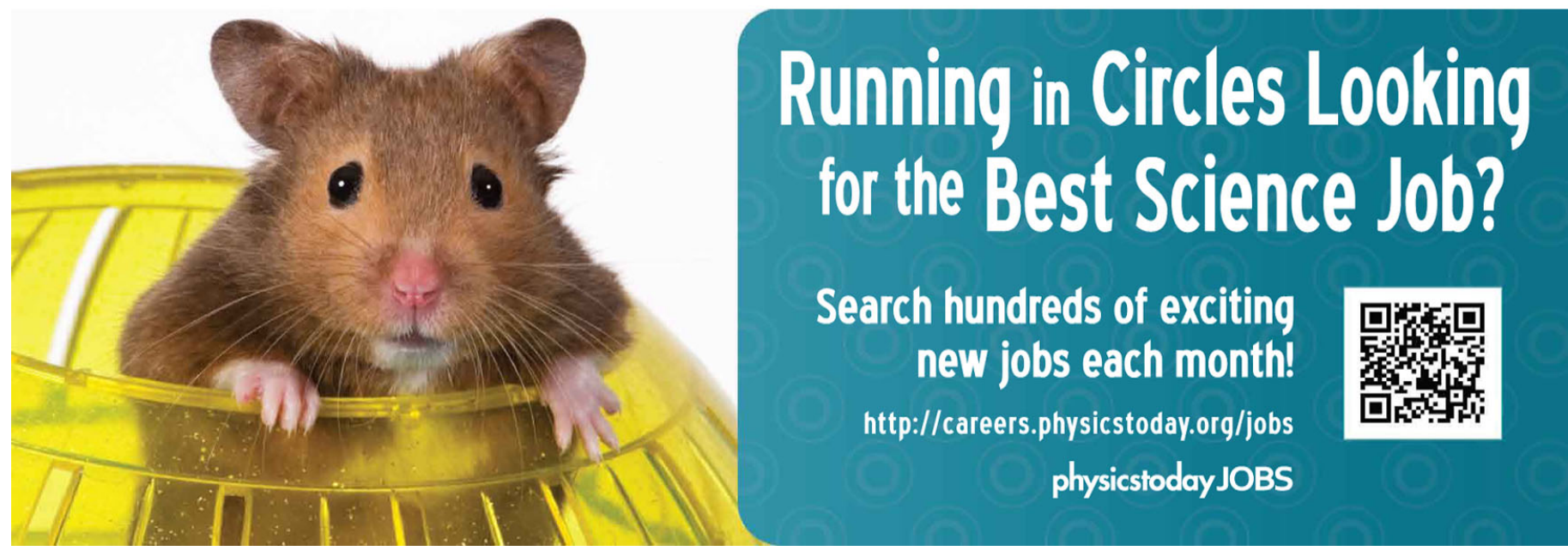




\title{
On Lagrangian single-particle statistics
}

\author{
Gregory Falkovich, ${ }^{1,2}$ Haitao Xu, ${ }^{1,3}$ Alain Pumir, ${ }^{1,4}$ \\ Eberhard Bodenschatz, ${ }^{1,3,5,6, a)}$ Luca Biferale, ${ }^{1,7}$ Guido Boffetta, ${ }^{1,8}$ \\ Alessandra S. Lanotte, ${ }^{1,9}$ and Federico Toschi ${ }^{1,10,11}$ \\ ${ }^{1}$ International Collaboration for Turbulence Research \\ ${ }^{2}$ Physics of Complex Systems, Weizmann Institute of Science, Rehovot 76100, Israel \\ ${ }^{3}$ Max Planck Institute for Dynamics and Self-Organization, D-37077 Göttingen, Germany \\ ${ }^{4}$ Laboratoire de Physique, Ecole Normale Supérieure de Lyon, 46, Université Lyon 1 \\ and CNRS, F-69007 Lyon, France \\ ${ }^{5}$ Institute for Nonlinear Dynamics, University of Göttingen, D-37077 Göttingen, Germany \\ ${ }^{6}$ Laboratory of Atomic and Solid State Physics and Sibley School of Mechanical \\ and Aerospace Engineering, Cornell University, Ithaca, New York 14853, USA \\ ${ }^{7}$ Department of Physics and INFN, University of Tor Vergata, Via della Ricerca Scientifica 1, \\ I-00133 Roma, Italy \\ ${ }^{8}$ Department of Physics and INFN, University of Torino, I-10125 Torino, Italy \\ ${ }^{9}$ ISAC-CNR, Str. Prov. Lecce-Monteroni, and INFN, Sezione di Lecce, I-73100 Lecce, Italy \\ ${ }^{10}$ Department of Physics, and Department of Mathematics and Computer Science, and J.M. \\ Burgerscentrum, Eindhoven University of Technology, 5600 MB Eindhoven, The Netherlands \\ ${ }^{11}$ IAC, CNR, Via dei Taurini 19, I-00185 Roma, Italy
}

(Received 23 January 2012; accepted 5 April 2012; published online 14 May 2012)

In turbulence, ideas of energy cascade and energy flux, substantiated by the exact Kolmogorov relation, lead to the determination of scaling laws for the velocity spatial correlation function. Here we ask whether similar ideas can be applied to temporal correlations. We critically review the relevant theoretical and experimental results concerning the velocity statistics of a single fluid particle in the inertial range of statistically homogeneous, stationary and isotropic turbulence. We stress that the widely used relations for the second structure function, $D_{2}(t) \equiv\left\langle[v(t)-v(0)]^{2}\right\rangle \propto \varepsilon t$, relies on dimensional arguments only: no relation of $D_{2}(t)$ to the energy cascade is known, neither in two- nor in three-dimensional turbulence. State of the art experimental and numerical results demonstrate that at high Reynolds numbers, the derivative $\frac{d D_{2}(t)}{d t}$ has a finite non-zero slope starting from $t \approx 2 \tau_{\eta}$. The analysis of the acceleration spectrum $\Phi_{A}(\omega)$ indicates a possible small correction with respect to the dimensional expectation $\Phi_{A}(\omega) \sim \omega^{0}$ but present data are unable to discriminate between anomalous scaling and finite Reynolds effects in the second order moment of velocity Lagrangian statistics. (ㅇ 2012 American Institute of Physics. [http://dx.doi.org/10.1063/1.4711397]

\section{INTRODUCTION}

From an elementary point of view, turbulent motion provides a way to dissipate energy in fluids, when forcing and effective dissipation act on vastly different scales. For energy to be transferred from the pumping scale to the dissipative scale, a cascade appears. Formal expression of the energy cascade is the exact flux law

$$
\left\langle(\delta \boldsymbol{u} \cdot \boldsymbol{r} / r)^{3}\right\rangle=-12 \varepsilon r / D(D+2),
$$

where $\delta \boldsymbol{u}$ is the Eulerian velocity difference (i.e., measured at the same time at points separated by $\boldsymbol{r}), \varepsilon$ is the mean energy dissipation/production rate and $D$ is the space dimensionality. Equation (1) is exact for the separation distance $r$ is in the so-called inertial range: $\eta \ll r \ll L$, where $\eta$ is the

\footnotetext{
a) Author to whom correspondence should be addressed. Electronic mail: eberhard.bodenschatz@ds.mpg.de.
} 
Kolmogorov length scale, the scale below which velocity field is smooth; $L$ is the integral length scale. For the Lagrangian velocity $\boldsymbol{v}(t)=\boldsymbol{u}(\boldsymbol{R}, t)$ with $\dot{\boldsymbol{R}}=\boldsymbol{v}$, the exact relation, which is a direct analog of the Eulerian flux law (1), is derived for the Lagrangian time derivative of the two-particle single-time velocity difference: $\left\langle d\left|\boldsymbol{v}\left(\boldsymbol{R}_{1}, t\right)-\boldsymbol{v}\left(\boldsymbol{R}_{2}, t\right)\right|^{2} / d t\right\rangle=-2 \varepsilon$ for $\left|\boldsymbol{R}_{1}-\boldsymbol{R}_{2}\right|$ in the inertial range. Let us remark that $\varepsilon$ has a sign depending on whether this is a direct $(\varepsilon>0$ for $D=3)$ or inverse $(\varepsilon<0$ for $D=2)$ cascade,${ }^{1-3}$ which follows from the fact that the main contribution to $d\left|\boldsymbol{v}\left(\boldsymbol{R}_{1}, t\right)-\boldsymbol{v}\left(\boldsymbol{R}_{2}, t\right)\right|^{2} / d t$ comes either from the force term for $D=2$ or from the dissipation acting at smaller scales for $D=3$.

The flux relation suggests the Kolmogorov-Obukhov scaling for the Eulerian velocity difference

$$
\left\langle\left((\delta \boldsymbol{u} \cdot \boldsymbol{r} / r)^{n}\right\rangle \simeq(\varepsilon r)^{n / 3} .\right.
$$

The general scaling law Eq. (2) is probably correct in two-dimensional space (2D). ${ }^{4,5}$ It is known to be incorrect in three-dimensional space (3D), and even though it provides a useful phenomenology for $n$ not very different from 3 , it is important that $\varepsilon$ does not determine the whole single-time statistics.

Here we ask whether the cascade idea can tell us anything about the Lagrangian statistics, i.e., temporal correlations of the single-particle velocity. It is tempting to relate the statistical properties of spatial and temporal velocity increments using the relation $r \simeq t \delta u(r)$. Since the velocity increment $\delta u(r)$ is a strongly fluctuating quantity whose statistics may depend on the scale $r$, using such casual arguments to establish relations between spatial and temporal statistics is questionable. The first attempt at deriving such a relation was made by Landau and Obukhov who simply assumed that the temporal change of the Lagrangian velocity is determined solely by the energy flux and suggested the relation $\delta \boldsymbol{v}(t)=\boldsymbol{v}(t ; \boldsymbol{R}(0))-\boldsymbol{v}(0, \boldsymbol{R}(0)) \simeq(\varepsilon t)^{1 / 2}$, which in particular suggests ${ }^{6}$

$$
D_{2}(t) \equiv\left\langle|\boldsymbol{v}(t)-\boldsymbol{v}(0)|^{2}\right\rangle \simeq \varepsilon t,
$$

for time lags $t$ in the Lagrangian inertial range: $\tau_{\eta} \ll t \ll T_{L}$, where $\tau_{\eta}=\left(\eta^{2} / \varepsilon\right)^{1 / 3}$ is the Kolmogorov time scale and $T_{L}=\left(L^{2} / \varepsilon\right)^{1 / 3}=L /\left\langle v^{2}\right\rangle^{1 / 2}$ is the large eddy turn over time.

We show below that the pure scaling of Eq. (3) does not follow from theoretical considerations, see Sec. II, and that it is not observed even at very large Reynolds numbers, see Sec. III where experimental and numerical data are presented.

\section{THEORETICAL CONSIDERATIONS}

\section{A. Time-irreversibility}

As a minor remark, we first note that since $\varepsilon$ in Eqs. (1) and (2) has a sign, Eq. (3) cannot contain $\varepsilon t$ but must rather use the positive quantity which is the energy injection rate $\langle\boldsymbol{f} \cdot \boldsymbol{u}\rangle$, where $\boldsymbol{f}(t)$ is the external forcing. Upon time reflection, both $t$ and $\langle\boldsymbol{f} \cdot \boldsymbol{u}\rangle$ change sign leaving Eq. (3) unchanged. More fundamental doubt on whether the flux can determine $\delta v(t)$ follows from the fact that the flux is a measure of irreversibility, while the single-particle Lagrangian statistics is time reversible. To prove the latter, note that the statistical homogeneity of the Eulerian velocities implies that the Lagrangian velocity $\boldsymbol{v}(t)=\boldsymbol{u}(\boldsymbol{R}(t), t)$ is statistically independent of the initial position $\boldsymbol{R}(0)$. If, additionally, the Eulerian velocity is statistically stationary, then so is the Lagrangian one. This follows by averaging the expectations involving $\boldsymbol{v}(t)$ over the initial position $\boldsymbol{R}(0)$ (on which they do not depend) and by the change of variables $\boldsymbol{R}(0) \mapsto \boldsymbol{R}(t)$ which leaves the velocity ensemble average invariant for incompressible flows. ${ }^{1,7}$ Consider now the time reversal $t \rightarrow-t$, $\boldsymbol{v} \rightarrow-\boldsymbol{v}$ and $\boldsymbol{v}(t)-\boldsymbol{v}(0) \rightarrow-\boldsymbol{v}(-t)+\boldsymbol{v}(0)$, this latter quantity turns into the initial one, $\boldsymbol{v}(t)-\boldsymbol{v}(0)$, after the time shift, which leaves the statistics invariant. This argument can be readily generalized to any correlation function explicitly invariant under galilean transformation, and involving only one Lagrangian point. Such correlation functions are expressible in terms of products of velocity differences of the velocity $v$ at different times, and are invariant under $t \rightarrow-t$. In contrast, the example involving two Lagrangian points cited in the introduction ${ }^{1-3}$ shows that measurements with more than one Lagrangian point permit to distinguish time irreversibility in the system. 


\section{B. Can velocity differences grow diffusively?}

Equation (3) suggests a diffusive growth of velocity difference. Indeed, diffusion is expected when $t$ exceeds the correlation time of the Lagrangian acceleration $\boldsymbol{a}(t)=d \boldsymbol{v} / d t$. One consequence would be that the probability density function (PDF) of single-particle Lagrangian velocity difference $\mathcal{P}(\delta \boldsymbol{v}, t)$ tends asymptotically to a Gaussian with increasing $t$, a result true for general random flows, not only for turbulence (with an energy cascade). The diffusivity $\lim _{t \rightarrow \infty}|\delta \boldsymbol{v}|^{2} / t$ generally has nothing to do with the energy flux even though it has the same dimensionality. In developed turbulence, the acceleration is known to be correlated on the large (integral) timescale $T_{L}$ (see also Eq. (5) and related discussion below). Since Eq. (3) is intended for time delays in the inertial interval, which is less than the acceleration correlation time, the PDF is then unlikely to have a self-similar form $\mathcal{P}(\delta \boldsymbol{v}, t) \propto F\left(|\delta \boldsymbol{v}|^{2} / \varepsilon t\right)$. Indeed, higher moments $D_{n}(t)$ all have an anomalous scaling. ${ }^{8-10}$ Especially, the data suggest that the exponents saturate at $\sim 2$, i.e., $\left\langle|\delta \boldsymbol{v}|^{n}\right\rangle \propto t^{2}$ for $n \rightarrow \infty$, which would be the probability that two independent events happen during time interval $t .{ }^{10}$ One can even imagine those two events being particle entering and exiting a vortex. ${ }^{9}$ We cannot think of any theoretical reason why the second moment should be special and have Kolmogorov scaling (as the third Eulerian moment does). Moreover, recall the reasoning that the second Eulerian velocity structure function in 3D does not have Kolmogorov scaling since a two-vector object already contains geometry. ${ }^{1}$ The same may be true for the second Lagrangian moment because it is really a two-particle object (since we consider statistics at the distances or time delays in the inertial interval).

\section{Energy considerations}

What experiments and numerics show, see Sec. III, is that $\left\langle|\delta \boldsymbol{v}|^{2}\right\rangle / \epsilon t=C(t)$ does not have a plateau but rather has a peak whose maximum slowly grows with the Reynolds number and whose width does not change much, see Figures 1 and 2. While the observed maximal values of $C(t)$ at the presently available Reynolds numbers are $6-7$ in 3D, they are very large (up to $O\left(10^{2}\right)$ ) in $2 \mathrm{D}$. These two observations: absence of a clear scaling and unexpectedly large value of this ratio, may in fact be intimately related. Indeed, if the relation in Eq. (3) made sense at least as an estimate all the way until $t \simeq T_{L}$, one can estimate the mean energy as $v^{2} \simeq C \varepsilon T_{L}$. For $3 \mathrm{D}$ case, $T_{L} \simeq L / v$ and we get $v^{3} \simeq C \varepsilon L$, which may be acceptable even with $C \simeq 6$. However, 2D numerics are done with a uniform friction term $-\alpha \boldsymbol{v}$ added to the Navier-Stokes equation; the turn over time of the largest vortex is of order $1 / \alpha$ so that decreasing $\alpha$ one increases the extent of the inverse cascade. The exact

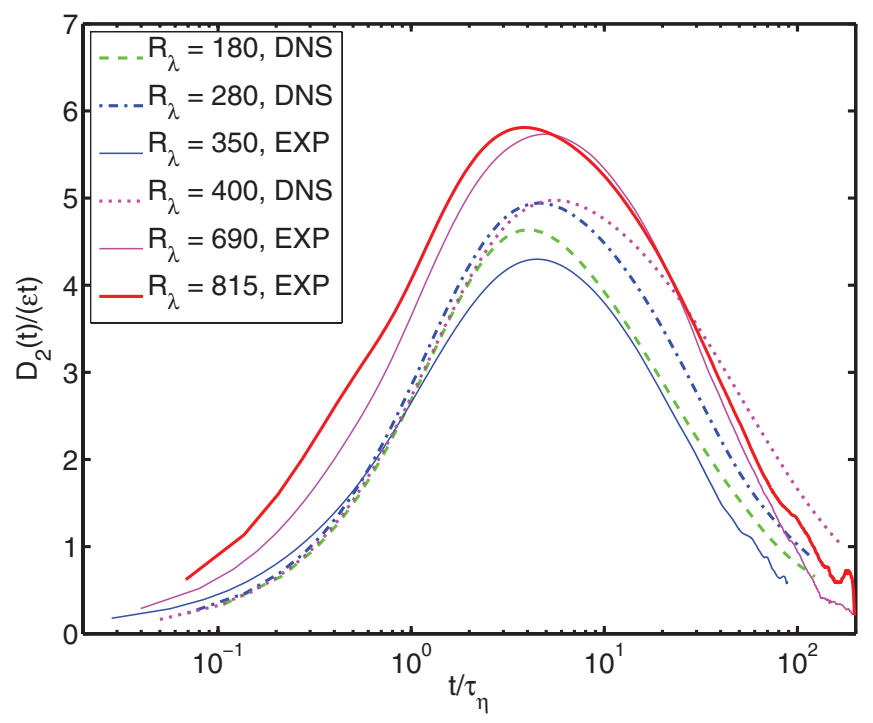

FIG. 1. Compensated second order Lagrangian structure function $D_{2}(t) /(\varepsilon t)$ obtained from 3D direct numerical simulations, ${ }^{9,11}$ labelled as "DNS," and particle tracking experiments, ${ }^{10,12}$ labelled as "EXP." 


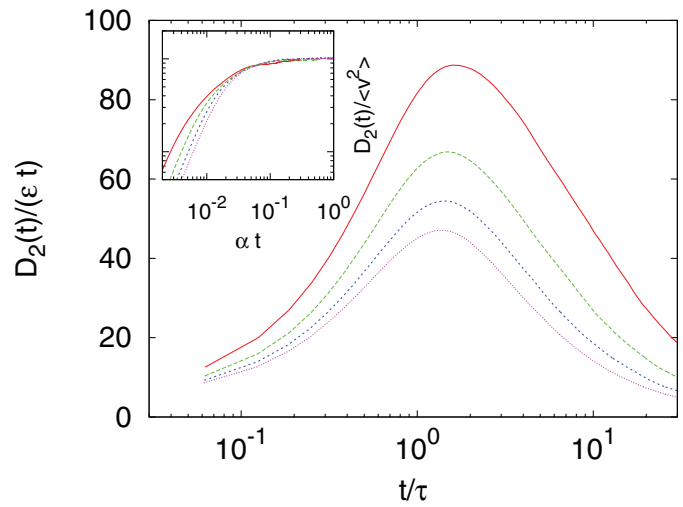

FIG. 2. Second order Lagrangian structure function $D_{2}(t)=\left\langle|\mathbf{v}(t)-\mathbf{v}(0)|^{2}\right\rangle$ for $2 \mathrm{D}$ simulation from $L / l_{f}=128(\alpha=0.02$, red continuous') to $L / l_{f}=16.9$ ( $\alpha=0.08$, pink dot-dashed) compensated with $\varepsilon t$. The Kolmogorov time $\tau_{\eta}$ is defined in terms of the enstrophy flux $\xi$ as $\tau_{\eta}=\xi^{-1 / 3}$. Inset: $D_{2}(t)$ compensated with mean square velocity $\left\langle v^{2}\right\rangle$ as a function of $\alpha t$.

conservation law in $2 \mathrm{D},\left\langle v^{2}\right\rangle=\varepsilon /(2 \alpha)$, means that Eq. (3) or similar law of growth cannot be valid all the way till $t \simeq 1 / \alpha$. This suggests that the Lagrangian correlation time is anomalously small, $T_{L} \simeq 1 /(C \alpha) \ll 1 / \alpha$, i.e., Lagrangian velocities decorrelate much faster than the large scale turn over time $1 / \alpha$ and that this time has little to do with the dynamical times induced by the flux in the cascade. The inset of Figure 2 shows that indeed $D_{2}(t)$ saturates at times much shorter than $1 / \alpha$.

\section{Fluctuation-dissipation theorem}

Another perspective on the Lagrangian pair correlation function can be given by the fluctuationdissipation theorem (FDT). Indeed, Lagrangian description of ideal flows is a canonical Hamiltonian description with Bernoulli invariant, $\int\left(v^{2} / 2+p\right) d \boldsymbol{r}$, as Hamiltonian. ${ }^{13}$ FDT states that at thermal equilibrium the detailed balance means that the time derivative of the pair correlation function of the coordinate is equal to the response function of the coordinate to the force. Since the "coordinate" conjugated to the force $\boldsymbol{f}$ is $\mathbf{v}$ then FDT would mean that the time derivative $d\left\langle v_{i}(0) v_{j}(t)\right\rangle / d t$ is equal to the response function $R_{i j}(t)=\left\langle\delta v_{i}(t) / \delta f_{j}(0)\right\rangle$. This response function can be found for a forcing delta-correlated in time, $\left\langle f_{i}(0) f_{j}(t)\right\rangle=\varepsilon P_{i j} \delta(t)$, we have $R_{i j}=\varepsilon \delta_{i j} H(t) / 2$, where $H(t)$ is the Heaviside function. In other words, if Eq. (3) were true with a proportionality factor unity, that would mean the detailed balance and FDT for a single-particle statistics, with $\varepsilon$ playing the role of temperature. This would suggest that the energy dissipation rate, $\varepsilon$, quantifies both the degree of irreversibility of two-particle statistics and play the role of temperature in the reversible singleparticle statistics, which seems surprising. Indeed, there is no reason to believe that FDT holds. In turbulence, each fluid particle is under the action of the pressure field created by all other particles: $d v_{j}(t) / d t=-\nabla_{j} p+f_{j}$, and it is entirely plausible that this coupling leads to a violation of FDT, which would manifest itself in the Lagrangian correlation function $\left\langle v_{i}(0) \nabla_{j} p(t)\right\rangle$. Notice that in this argument we do not distinguish 2D and 3D; while the forcing has different spatial correlations in these two cases (short- and long-correlated, respectively), what matters for the single particle is the temporal correlations of the force, which may be similar.

\section{CONSISTENCY WITH OBSERVATIONS FROM DATA}

\section{A. Acceleration auto-correlation}

Both from fundamental FDT perspective and in order to check the linear relation between $D_{2}$ and $t$, Eq. (3), or equivalently, the existence of a plateau in the variable $D_{2}(t) / t$ from experimental or numerical data, it is useful to relate the structure function $D_{2}$ to the acceleration auto-correlation 
function. The kinematic relation

$$
\begin{aligned}
\frac{d}{d t}\left\langle[\boldsymbol{v}(t)-\boldsymbol{v}(0)]^{2}\right\rangle & =2\langle\boldsymbol{a}(t) \cdot[\boldsymbol{v}(t)-\boldsymbol{v}(0)]\rangle \\
& =2 \int_{0}^{t}\left\langle\boldsymbol{a}(0) \cdot \boldsymbol{a}\left(t^{\prime}\right)\right\rangle d t^{\prime}
\end{aligned}
$$

implies that a scaling of $\left\langle[\boldsymbol{v}(t)-\boldsymbol{v}(0)]^{2}\right\rangle=C \varepsilon t$ requires that the Lagrangian acceleration autocorrelation $\langle\boldsymbol{a}(0) \cdot \boldsymbol{a}(\tau)\rangle$ is zero in the scaling range while the integral $\int_{0}^{t}\langle\boldsymbol{a}(0) \cdot \boldsymbol{a}(\tau)\rangle d \tau$ gives the constant $C \varepsilon / 2$. However, it is well known that in a statistically stationary flow fluid, the Lagrangian acceleration autocorrelation has to satisfy the constraint ${ }^{14}$

$$
\int_{0}^{\infty}\langle\boldsymbol{a}(0) \cdot \boldsymbol{a}(t)\rangle d t=0
$$

which implies that the right-hand side in Eq. (4) is unlikely to be constant, thus pointing to limitations of the linear relation $D_{2}(t) \propto \varepsilon t$. In fact, the available experimental ${ }^{15}$ and numerical ${ }^{16,17}$ data show that the acceleration autocorrelation first decreases monotonically, crosses zero at $2 \tau_{\eta}$, then slowly relaxes back to zero at large time lags. The scaling range of $\left\langle[v(t)-\boldsymbol{v}(0)]^{2}\right\rangle$ is usually identified at the peak of $\langle\boldsymbol{a}(t) \cdot[\boldsymbol{v}(t)-\boldsymbol{v}(0)]\rangle$, which is associated with the zero-cross time of the acceleration autocorrelation, i.e., at $2 \tau_{\eta}$. As shown in Fig. 3, experimental and numerical data do not support a plateau of $\frac{d}{d t}\left\langle(\boldsymbol{v}(t)-\boldsymbol{v}(0))^{2}\right\rangle$ around its peak value, a fact directly related to the shape of the acceleration autocorrelation.

\section{B. Acceleration spectra}

The practical difficulty in observing any "inertial range" scaling property from the function $\left\langle|\boldsymbol{v}(t)-\boldsymbol{v}(0)|^{2}\right\rangle$, related to the small range of scales $T_{L} / \tau_{\eta} \approx 0.08 \times R_{\lambda}$ has been already noticed several times. ${ }^{18,19}$ Even so, one may wonder why it turns out to be so difficult to extract any strong evidence for any scaling directly from the structure function. In comparison, the data obtained in the Fourier domain, in particular, concerning the spectrum of acceleration, $\Phi_{A}(\omega)$, suggest a scaling range compatible with $\Phi_{A}(\omega) \sim \omega^{0}$ for $1 / T_{L} \lesssim \omega \lesssim 1 / \tau_{\eta},{ }^{19,20}$ possibly up to small corrections which we will discuss later. The observation of a spectrum $\Phi_{A}(\omega) \sim \omega^{0}$ is generally consistent with the narrowly peaked acceleration correlation function. ${ }^{17}$ Viewed over a scale $t \sim T_{L}$, this spike becomes infinitely thin as the ratio $T_{L} / \tau_{\eta} \rightarrow \infty$. On the other hand, the spectrum behaves, for $\omega T_{L} \rightarrow 0$, as $\Phi_{A}(\omega) \sim\left(\omega T_{L}\right)^{2}$, in agreement with the fact that the correlation function of velocity decreases essentially exponentially: ${ }^{8}\langle\mathbf{v}(0) \cdot \mathbf{v}(t)\rangle \sim \exp \left(-t / T_{L}\right)$, except for finite values of the "inner variable" $t / \tau_{\eta}{ }^{19}$ This is consistent with our own observation, see Fig. 3(b), that as the Reynolds number increases, the correlation function $\langle\mathbf{a}(t) \cdot \delta \mathbf{v}(t)\rangle$ becomes increasingly cusped, exhibiting a slope discontinuity, when plotted as a function of the outer variable, $t / T_{L}$. In fact, the existence of a plateau of $\langle\mathbf{a}(t) \cdot(\mathbf{v}(t)-\mathbf{v}(0))\rangle$, equivalent to the linear relation Eq. (3), may seem a bit contrived, since the outer variable does not show any trace of a plateau at all. While this fact does not in itself completely preclude the existence a scaling range, it indeed restricts its application to an even smaller range of time scales than the already quite restricted range $\tau_{\eta} \ll t \ll T_{L}$. Thus, aside from its uncertain status as a manifestation of Kolmogorov theory, the existence of a linear scaling of the structure function, Eq. (3), is in practice very difficult to measure, due to the functional form of the acceleration correlation function. The stochastic model developed in Refs. 19 and 21 shows that a decade of plausibly constant value of $\langle\mathbf{a}(t) \cdot(\mathbf{v}(t)-\mathbf{v}(0))\rangle$ requires a Reynolds number of $R_{\lambda} \sim 10^{4}$, which is the highest Reynolds number that can be achieved in laboratory experiments in the foreseeable future.

Possible evidence for a deviation from the linear behavior of Eq. (3) are provided by the spectrum $\Phi_{A}(\omega)$. The recently published data ${ }^{19}$ show a weak deviation from the $\omega^{0}$ scaling, and may be plausibly fitted with a small exponent,

$$
\Phi_{A}(\omega) \approx A_{0} \varepsilon\left(\omega T_{L}\right)^{\mu}
$$




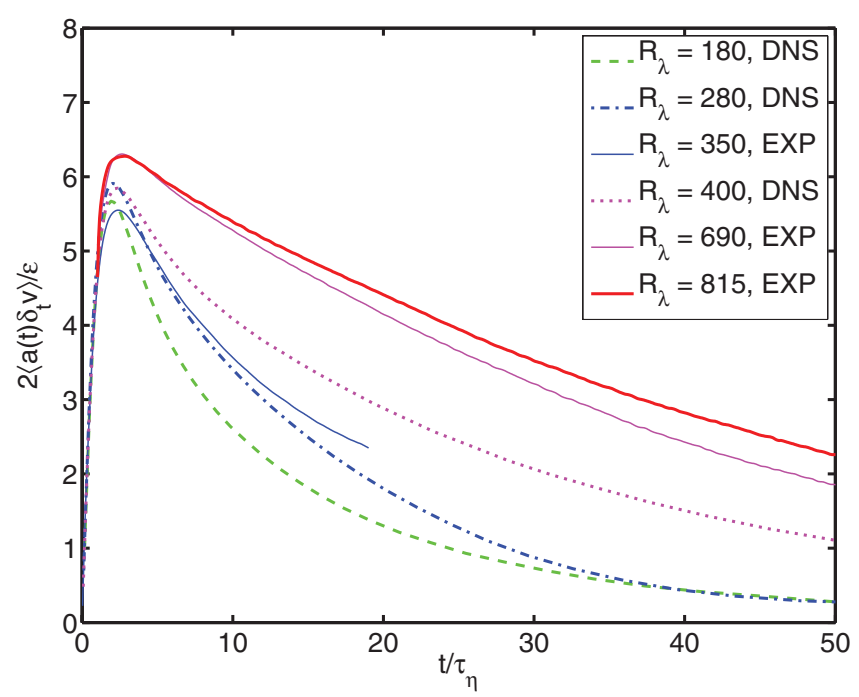

(a)

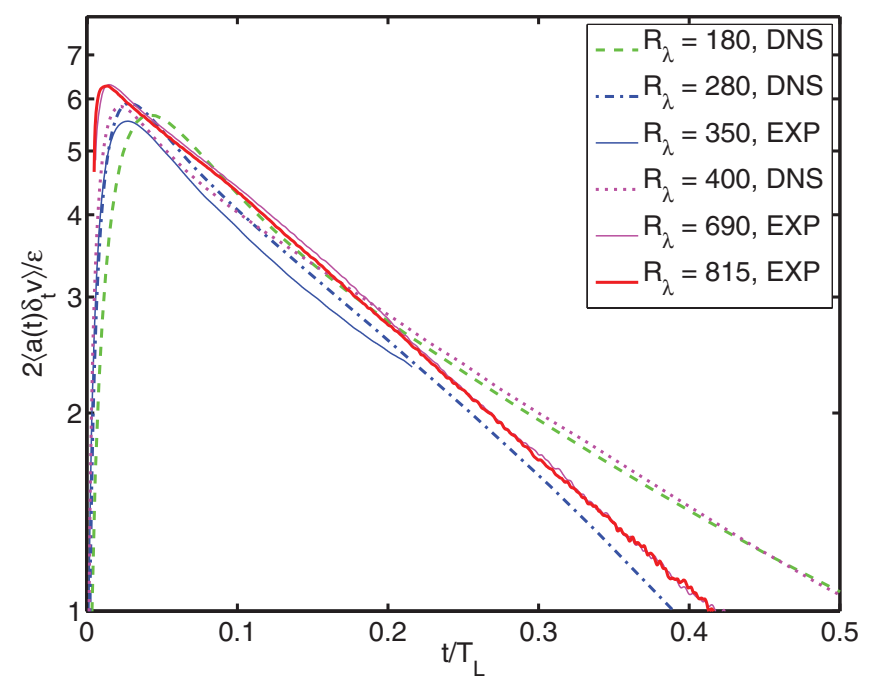

(b)

FIG. 3. Derivative of the second order velocity structure function, $\frac{d}{d t}\left\langle[\boldsymbol{v}(t)-\boldsymbol{v}(0)]^{2}\right\rangle=2\langle\boldsymbol{a}(t) \cdot[\boldsymbol{v}(t)-\boldsymbol{v}(0)]\rangle$ plotted versus $\tau / \tau_{\eta}$ in (a) and $\tau / T_{L}$ in (b). Data are derived from $D_{2}(t)$ as plotted in Fig. 1.

for $1 / T_{L} \lesssim \omega \lesssim 1 / \tau_{\eta}$, with $\mu \approx 0.12-0.14$. The very good collapse of the spectra in Fig. 5(a) of Ref. 19, suggests that the value of $A_{0}$ is effectively independent of the Reynolds number, at least in the range covered in their studies. We first remark that this weak deviation from a flat spectrum would result in a small modification to the linear scaling of Eq. (3),

$$
\left\langle|\boldsymbol{v}(t)-\boldsymbol{v}(0)|^{2}\right\rangle \simeq t^{1-\mu}
$$

We also note that for consistency the integral of the spectrum has to be equal to the acceleration variance

$$
\left\langle\mathbf{a}^{2}\right\rangle=\int_{0}^{\infty} \Phi_{A}(\omega) d \omega
$$


In the limit $T_{L} / \tau_{\eta} \rightarrow \infty$, the integral in Eq. (8), using the functional form Eq. (6), yields

$$
\left\langle\mathbf{a}^{2}\right\rangle \approx A_{0} \varepsilon \frac{\varepsilon}{\tau_{\eta}}\left(\frac{T_{L}}{\tau_{\eta}}\right)^{\mu} \approx A_{0} \varepsilon \frac{\varepsilon^{3 / 2}}{v^{1 / 2}}\left(\frac{T_{L}}{\tau_{\eta}}\right)^{\mu} .
$$

Interestingly, Eq. (9) suggests that the ratio $\left\langle a^{2}\right\rangle /\left(\varepsilon^{3 / 2} v^{-1 / 2}\right)$ grows with $R_{\lambda} \cdot{ }^{19,22,23}$ Alternatively, we note that a drift of the upper limit of the energetic band of frequency, as proposed in Ref. 19, may also explain the Reynolds dependency of the acceleration variance. This demonstrates that the available data, obtained at the highest available Reynolds number, do not unambiguously support the widely accepted scaling relation Eq. (3). As pointed out already, a systematic study of the acceleration spectrum appears much more promising than a direct study of the structure function.

\section{DISCUSSION AND PERSPECTIVE}

Let us also remark that interpolation schemes encompassing viscous, inertial, and large-scales properties have been used to study both Eulerian ${ }^{24,25}$ and Lagrangian quantities. ${ }^{26}$ Although these are convenient tools to describe the scaling properties of $D_{2}(t),{ }^{27}$ they do not provide new physical information as the scaling relations are prescribed while constructing the interpolation formulae.

For the reasons described above, it is clear that the study of the single-particle Lagrangian statistics needs some benchmark that is different from Eq. (3). The uncertain status of Eq. (3) implies that the use of extended self-similarity ${ }^{28}$ to study the moments of $\delta \boldsymbol{v}$ with $\left\langle|\delta \boldsymbol{v}|^{2}\right\rangle$ as a normalization is questionable, contrary to the spatial case, where the exact relation Eq. (1) holds. Comparison between energy cascades in 2D (Ref. 29) and 3D, enstrophy cascade, elastic turbulence, and other cases is of much interest. It would also be interesting to study multiple multi-time, multipoint correlation functions ${ }^{30,31}$ and see how Lagrangian and Eulerian correlations are related, ${ }^{32}$ both in the case of two-dimensional turbulence, ${ }^{29}$ where available evidence does not suggest any Eulerian intermittency, and of three-dimensional turbulence.

One of the hallmarks of fluid turbulence is the breakdown of time reversibility whose measure is the flux. The flux also determines the level of turbulence and thus must influence the Lagrangian statistics as well. Since the single-particle Lagrangian statistics is time-reversible, it is yet unclear how exactly moments $\left\langle(\boldsymbol{v}(t)-\boldsymbol{v}(0))^{n}\right\rangle$ depend on $\epsilon$ and whether there is a clear scaling with $t$.

\section{ACKNOWLEDGMENTS}

This work was initiated while we were attending the "The nature of turbulence" 2011 program at the Kavli Institute for Theoretical Physics (KITP), UCSB and was supported in part by the US National Science Foundation under Grant No. NSF PHY05-51164. The revision of the manuscript was finished during the "New directions in turbulence" 2012 program at the Kavli Institute for Theoretical Physics China at the Chinese Academy of Science (KITPC). We also acknowledge partial support from the COST Action MP0806 "Particles in turbulence".

${ }^{1}$ G. Falkovich, K. Gawedzki, and M. Vergassola, "Particles and fields in fluid turbulence," Rev. Mod. Phys. 73, 913-975 (2001).

${ }^{2}$ R. J. Hill, "Equations relating structure functions of all orders," J. Fluid Mech. 434, 379-388 (2001).

${ }^{3}$ A. Pumir, B. Shraiman, and M. Chertkov, "The Lagrangian view of energy transfer in turbulent flow," Europhys. Lett. 56, 379-385 (2001).

${ }^{4}$ R. Kraichnan, "Inertial ranges in two-dimensional turbulence," Phys. Fluids 10, 1417 (1967).

${ }^{5}$ G. Boffetta and R. Ecke, “Two-dimensional turbulence," Annu. Rev. Fluid Mech. 44, 427 (2012).

${ }^{6}$ L. Landau and E. Lifshitz, Fluid Mechanics (Pergamon, London, 1959).

${ }^{7}$ A. S. Monin and A. M. Yaglom, Statistical Fluid Mechnics (MIT, Boston, 1972).

${ }^{8}$ N. Mordant, P. Metz, O. Michel, and J.-F. Pinton, "Measurement of Lagrangian velocity in fully developed turbulence," Phys. Rev. Lett. 87, 214501 (2001).

${ }^{9}$ L. Biferale, G. Boffetta, A. Celani, A. Lanotte, and F. Toschi, "Particle trapping in three-dimensional fully developed turbulence," Phys. Fluids 17, 021701 (2005).

${ }^{10} \mathrm{H}$. Xu, M. Bourgoin, N. Ouellette, and E. Bodenschatz, "High order Lagrangian velocity statistics in turbulence," Phys. Rev. Lett. 96, 024503 (2006).

${ }^{11}$ J. Bec, L. Biferale, A. Lanotte, A. Scagliarini, and F. Toschi, “Turbulent pair dispersion of inertial particles,” J. Fluid Mech. 645, 497-528 (2010). 
${ }^{12}$ H. Xu, N. T. Ouellette, D. Vincenzi, and E. Bodenschatz, "Acceleration correlations and pressure structure functions in high-Reynolds number turbulence," Phys. Rev. Lett. 99, 204501 (2007).

${ }^{13}$ G. Falkovich, Fluid Mechanics, A Short Course for Physicists (Cambridge University Press, 2011).

${ }^{14} \mathrm{~T}$. Tennekes and J. L. Lumley, A First Course in Turbulence (MIT, Cambridge, MA, 1972).

${ }^{15}$ N. Mordant, A. M. Crawford, and E. Bodenschatz, "Three-dimensional structure of the Lagrangian acceleration in turbulent flows," Phys. Rev. Lett. 93, 214501 (2004).

${ }^{16} \mathrm{P}$. Yeung and S. Pope, "Lagrangian statistics from direct numerical simulations of isotropic turbulence," J. Fluid Mech. 207, 531-586 (1989).

${ }^{17}$ P. Yeung, S. Pope, E. Kurth, and A. Lamorgese, "Lagrangian conditional statistics, acceleration and local relative motion in numerically simulated isotropic turbulence," J. Fluid Mech. 582, 399-422 (2007).

${ }^{18}$ J. Hackl, P. Yeung, and B. Sawford, "Multi-particle and tetrad statistics in numerical simulations of turbulent dispersion," Phys. Fluids 23, 065103 (2011).

${ }^{19}$ B. Sawford and P. Yeung, "Kolmogorov similarity scaling for one-particle Lagrangian statistics," Phys. Fluids 23, 091704 (2011).

${ }^{20}$ R.-C. Lien and E. A. D'Asaro, "The Kolmogorov constant for the Lagrangian velocity spectrum and structure function," Phys. Fluids 14, 4456-4459 (2002).

${ }^{21}$ B. L. Sawford, "Reynolds number effects in Lagrangian stochastic models of turbulent dispersion," Phys. Fluids A 3 , 1577-1586 (1991).

${ }^{22}$ P. Yeung, S. Pope, A. Lamorgese, and D. Donzis, "Acceleration and dissipation statistics of numerically simulated isotropic turbulence," Phys. Fluids 18, 065103 (2006).

${ }^{23}$ G. Gulitski, M. Kholmyansky, W. Kinzelbach, B. Lüthi, A. Tsinober, and S. Yorish, "Velocity and temperature derivatives in high-Reynolds-number turbulent flows in the atmospheric surface layer. Part 2. Accelerations and related matters," J. Fluid Mech. 589, 83-102 (2007).

${ }^{24}$ C. Meneveau, "Transition between viscous and inertial-range scaling of turbulence structure functions," Phys. Rev. E 54, 3657-3663 (1996)

${ }^{25}$ S. Kurien and K. Sreenivasan, "Anisotropic scaling contributions to high-order structure functions in high-reynolds-number turbulence,” Phys. Rev. E 62, 2206-2212 (2000).

${ }^{26}$ A. Arneodo et al., "Universal intermittent properties of particles trajectories in highly turbulent flows," Phys. Rev. Lett. 100, 254504 (2008)

${ }^{27}$ L. Biferale and A. Lanotte, "About the second order moment of Lagrangian velocity increments," University of California Santa Barbara, Kavli Institute Preprint, NSF-ITP-11-103, 2011.

${ }^{28}$ R. Benzi, S. Ciliberto, R. Tripiccione, C. Baudet, F. Massaioli, and S. Succi, "Extended self-similarity in turbulent flows," Phys. Rev. E, 48, R29-R32 (1993).

${ }^{29}$ O. Kamps and R. Friedrich, "Lagrangian statistics in forced two-dimensional turbulence," Phys. Rev. E 78, 036321 (2008).

${ }^{30}$ D. Mitra and R. Pandit, "Varieties of dynamic multiscaling in fluid turbulence," Phys. Rev. Lett. 93, 024501 (2004).

${ }^{31}$ L. Biferale, E. Calzavarini, and F. Toschi, "Multi-time multi-scale correlation functions in hydrodynamic turbulence," Phys. Fluids 23, 085107 (2011).

${ }^{32}$ O. Kamps, R. Friedrich, and R. Grauer, "Exact relation between Eulerian and Lagrangian velocity increment statistics," Phys. Rev. E 79, 066301 (2009). 